\title{
IDENTIFICATION AND CHARACTERIZATION OF A HUMAN TRANSTHYRETIN VARIANT
}

\author{
John R. Strahler ${ }^{1}$, Barnett B. Rosenblum ${ }^{1,2}$, and Samir M. Hanash ${ }^{1}$
}

\author{
${ }^{1}$ Department of Pediatrics, University of Michigan, \\ Ann Arbor. MI 48109 \\ 2 Department of Pediatrics. University of Illinois, \\ College of Medicine at Chicago. Chicago, Illinois 60612
}

Received September 8, 1987

SUMMARY: An apparent $\mathrm{Mr}$ variant of plasma transthyretin (TTR), previously detected using 2-D PAGE, is the first reported occurrence of this type of human TTR variant. We characterized the variant TTR to determine the nature of this difference. Comparative tryptic peptide maps of variant and normal TTR and sequencing of peptides which differed indicated the variant contained a single amino acid substitution of valine for tyrosine at position 116. Because such a change requires two nucleotide substitutions, we postulate the variant arose through mutation in codon 116 of a heretofore unrecognized polymorphic or rare variant allele of TTR. 1987 Academic Press, Inc.

In the course of our studies of genetic variation of plasma proteins in 56 Caucasoid families, an apparent molecular weight variant of transthyretin (TTR, formerly thyroxine-binding prealbumin) was discovered (1). The variant appeared in two generations in healthy adult individuals in a family of French Canadian descent. Other reports of human genetic variation in TTR have appeared. Asakawa et al. (2) detected one electrophoretic variant which was more cathodal than normal TTR in a study of 109 Japanese families. Altland et al.(3) have detected 3 different electrophoretic variants of TTR in the German population. Two had pI's more basic and one more acidic than normal TTR. One of the basic variants was observed in 7 families and the other two variants were observed each in a single family in a study of 4,000 non-related families. Lalloz et al. (4) have detected a genetic variant TTR which has increased affinity for $\mathrm{T}_{4}$ but which is indistinguishable from normal TTR by both isoelectric focusing and SDS PAGE. To our knowledge, the only reported instances of genetic variation in TTR for which the amino acid substitution has been identified have been in individuals affected with familial amvloidotic polvneuropathy (FAP). In type I FAP of Japanese, Portuguese, Swedish and Greek origins the genetic lesion has been identified as a valine to methionine substitution at position 30 of TTR (5-8). In the case of type I FAP of Jewish origin, the amino acid substitution is isoleucine for 
phenylalanine at position 33 (9). Type II FAP of Indiana/Swiss origin has a serine for isoleucine at position $84(10)$ and the Appalachian type has alanine for threonine at position 60 (11).

The current investigation describes the characterization of the apparent $\mathrm{M}_{\mathrm{r}}$ variant of TTR which we have detected. In addition we describe the implementation of a purification procedure at the nanomole level which is useful when sample size is limiting.

\section{MATERIALS AND METHODS}

Materials: Serum or plasma was prepared from proband (AP) peripheral blood following informed consent. Human plasma transthyretin (CalBiochem) was used as a control.

Purification of TTR: TTR was isolated from serum of the proband (AP), heterozygous for TTR, by anion exchange HPLC essentially as previously described (12). AP serum (1.3 $\mathrm{ml}$ containing $\sim 10 \mathrm{nmole}$ TTR) was injected on an AX300 column $(10 \mathrm{~m} \mu, 4 \times 100 \mathrm{~mm}$. Synchrome, Linden, IN) equilibrated with $4 \mathrm{mM} \mathrm{Na}$ phosphate, $\mathrm{pH} 7.8$. Protein was eluted with a $\mathrm{Na}$ phosphate gradient as described in the legend to Figure 2. TTR was identified by double immunodiffusion using rabbit antibody to human TTR (CalBiochem) and verified by 2D-PAGE. Fractions containing TTR were pooled, diluted with an equal volume of deionized water and chromatographed a second time on an AX300 column except that the buffers were adjusted to $\mathrm{pH} 7.0$. TTR was further purified by affinity chromatography on Aff-Gel Blue to remove the bulk of contaminating albumin and retinol-binding protein (7). TTR containing fractions were pooled. dialyzed against water and lyophilized. Protein was reconstituted with phosphate buffered saline (PBS) and separated on a gel permeation column (I-125, $7 \mathrm{x}$ $250 \mathrm{~mm}$, Waters) equilibrated with PBS at a flow rate of $0.7 \mathrm{ml} / \mathrm{min}$. TTR was further purified by reverse phase HPLC on a C4 column (10 $\mathrm{m} \mu, 4 \times 100 \mathrm{~mm}$, Vydac). The reverse phase column was equilibrated with $0.1 \%$ trifluoroacetic acid (TFA) in water. Protein was eluted with a $0.05 \%$ TFA in MeCN gradient as described in Figure 3.

2-D polyacrylamide gel electrophoresis: Two-dimensional polyacrylamide gel electrophoresis of plasma and HPLC column effluent fractions was as previously described (13). Plasma 2-D gels were stained with Coomassie Brilliant Blue R-250 for screening for TTR variants. 2-D gels of HPLC column effluent fractions were silver stained by the method of Merril el al. (14). Immunoblots on nitrocellulose were a modification of the method of Burnett (15) as previously described (16). First antibody was rabbit anti-TTR or rabbit anti-retinol-binding protein (CalBiochem). Second antibody was horseradish peroxidase conjugated goat anti-rabbit IgG (Sigma). 4-chloro1-naphthol was used as substrate.

Tryptic peptide mapping: Normal TTR and AP-TTR were treated with 70\% formic acid for $12 \mathrm{hr}(9)$ at room temperature and lyophilized. Each was then digested with trypsin (Miles) treated with L-1-tosylamido-2-phenylethyl chloromethyl ketone (TPCK) in $0.2 \mathrm{M}$ ammonium bicarbonate, $\mathrm{pH} 8.2$, for $2 \mathrm{hr}$ at $37^{\circ}$ (enzyme/substrate, $\mathrm{E} / \mathrm{S}$, ratio $=1 / 50$, w/ w). A second aliquot of TPCK-trypsin was added and the digestion continued for an additional $18 \mathrm{hr}$ (final E $/ \mathrm{S}$ ratio $=1 / 25, \mathrm{w} / \mathrm{w}$ ). Digests were acidified with glacial acetic acid, lyophilized, and peptides separated by reverse phase HPLC on a column of C18 $\mu$ Bondapak (Waters) as described in the legend to Figure 4. Peptides not completely resolved were further chromatographed on the same column using isopropanol/MeCN (1/ $2, \mathrm{v} / \mathrm{v}$ ) in place of $\mathrm{MeCN}$.

Amino acid analysis and sequence determination: Amino acid analyses were performed as described by Tarr (17) for PTC-amino acids separated by reverse phase HPLC. 
Sequence analyses of selected peptides were by the manual sequence film method with polybrene of Tarr (17). Occasionally, a gas phase sequencer (Applied Biosystems) was used for extended sequences. PTH-derivatives were identified by reverse phase HPLC, on an Ultrasphere ODS column developed with a step gradient in $88 \mathrm{mM}$ ammonium acetate, $\mathrm{pH} 4.9$, of 25 to $51 \% \mathrm{MeCN}$ (17). Occasionally, identification of a PTH-amino acid was confirmed by chromatography on a Nova-Pak C18 column developed with a gradient of $100 \mathrm{mM} \mathrm{K}$ phosphate vs $84 \% \mathrm{MeCN}$ as previously described (17).

\section{RESULTS AND DISCUSSION}

A section of a 2-D gel of normal plasma and AP-plasma, from an individual heterozygous for the TTR variant, is shown in Figure 1A. Normal TTR appears as two spots of equal $M_{r}$, the more anodic form being much less intense. The variant TTR is seen as having a higher apparent molecular weight than normal TTR. The variant is present in slightly reduced amounts relative to normal TTR, both on silver stained gels (Figure 1A) and Coomassie Blue stained gels (not shown). The corresponding region of an immunoblot. probed with anti-TTR, is shown in Figure 1B. Both normal and variant spots react with anti-TTR with approximately equal intensity after peroxidase staining. No other polypeptides cross-react with anti-TTR.

Initial fractionation of serum by anion exchange HPLC (Figure 2A) resulted in the elution of TTR in two peaks, the first representing the TTR tetramer and the second the TTR-retinol binding protein (RBP) complex. Both fractions contained normal and variant TTR as determined by 2-D PAGE of individual fractions. TTR containing fractions were pooled, diluted with water and chromatographed a second time on an anion exchange HPLC column (Figure $2 \mathrm{~B}$ and $2 \mathrm{C}$ ) using a modified buffer $\mathrm{pH}$ and gradient to remove additional albumin. TTR was again eluted in two peaks (TTR tetramer and TTR-RBP complex, Figure 2C), each containing normal and variant TTR. Affinity chromatography on AffiGel Blue was then performed to remove RBP and any remaining albumin (not shown). After lyophilization and reconstitution of the sample. the TTR was applied to a gel permeation column. The TTR containing peak comprised

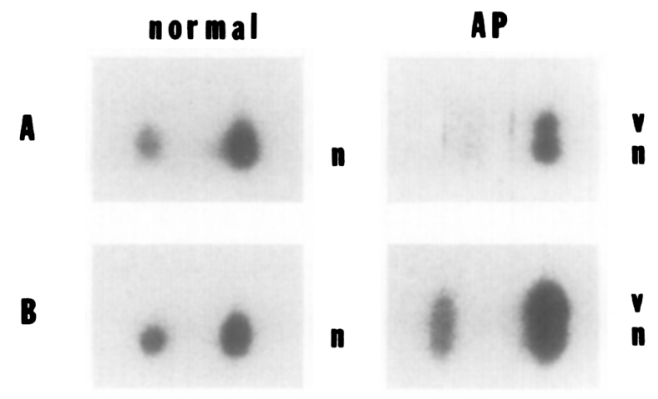

Figure 1. A section of a 2-D gel of normal and AP plasma. The normal $(N)$ and variant (V) TTR on (A) silver stained gels and (B) immunoblots with anti-TTR. 

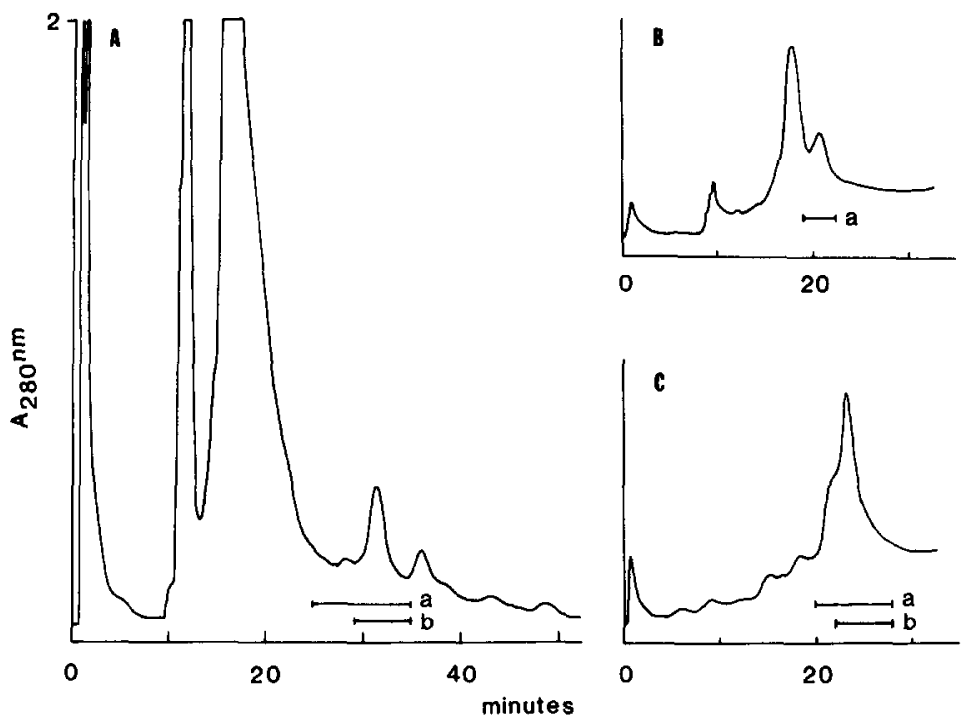

Figure 2. Anion exchange HPLC of AP serum. (A) AP serum (1.3ml) was injected on an AX300 column equilibrated with buffer A: $4 \mathrm{mM}$ sodium phosphate, pH7.8. Buffer B: $0.4 \mathrm{M}$ sodium phosphate, $\mathrm{pH} 7.8$. Gradient: $0-30 \% \mathrm{~B}$ in 30 min then $30-60 \% \mathrm{~B}$ in 10 min. Flow rate: $0.7 \mathrm{ml} / \mathrm{min}$. Bars indicate fractions immunoreactive with anti-TTR (a) or with anti-RBP (b). (B) and (C) Rechromatography of fractions immunoreactive to anti-TTR only or to anti-TTR and anti-RBP, respectively. Buffers $A$ and $B$ were adjusted to $\mathrm{pH} 7.0$. Gradient: $10-30 \% \mathrm{~B}$ in $30 \mathrm{~min}$.

$50 \%$ of the $280 \mathrm{~nm}$ absorbing material and contained both normal and variant TTR (not shown). AP-TTR was further purified by reverse phase HPLC on a $\mathrm{C}_{4}$ column (Vydac), using TFA as ion pairing agent and development with a MeCN gradient (Figure 3). Normal and variant TTR's were co-purified through this step and appeared homogeneous as judged by 2-D electrophoresis.

Purified AP-TTR, containing both normal and variant TTR, was digested with trypsin. Tryptic peptides were separated by reverse phase HPLC on a $\mathrm{C}_{18} \mu$ Bondapak column (Figure 4). Two new peptides, T11-12-13* and T12-13*, were found in the tryptic peptide map of AP-TTR (bottom) compared to that of normal TTR (top). In addition, the corresponding normal peptide was present in reduced amount in the mixture and in approximately the same amount as the variant peptide. Yields of individual tryptic peptides were low due to the extensive $\beta$ pleat structure of TTR which confers substantial resistance to the protein to tryptic digestion (7). Fifty picomole of the variant and normal peptides T11-12-13 were obtained for microsequence analysis. Reliable amino acid sequence information was obtained for the first 13 cycles of Edman degradation. By comparison with the published sequence for TTR (18), both peptides were identified as beginning at residue 104 in the C-terminal region of TTR (127 total residues). An amino acid difference was found at cycle 13 (residue 116) where the normal peptide had a tyrosine and the variant peptide had a valine. Such an amino 


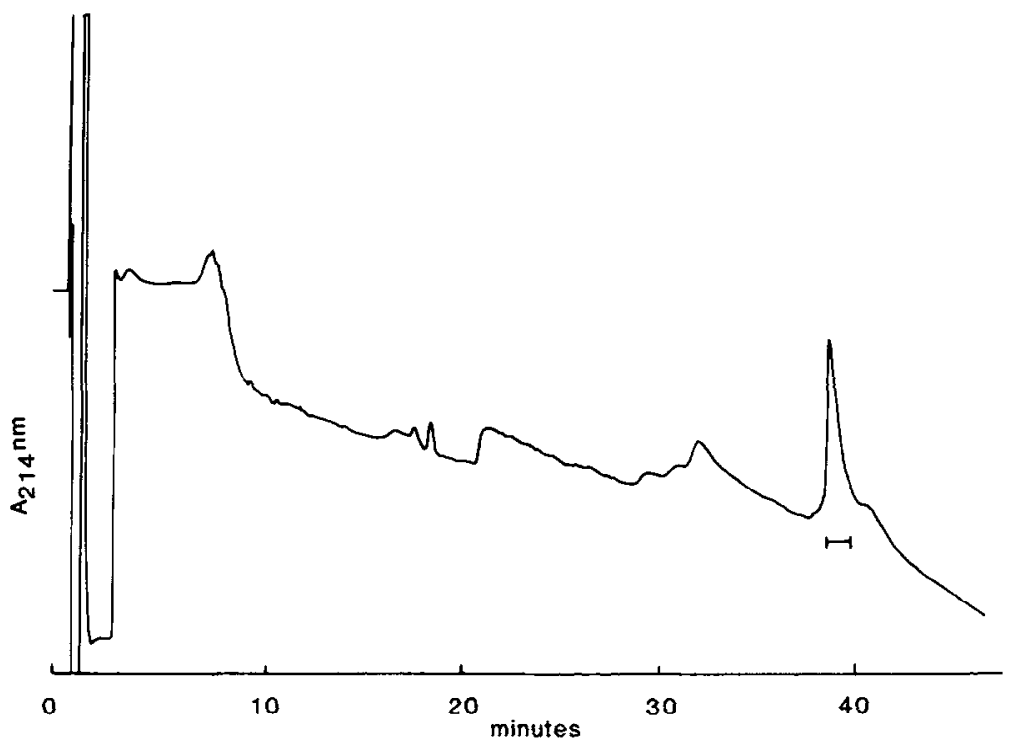

Figure 3. Reverse phase HPLC of AP.TTR from the gel permeation step. Column: Vydac C4 equilibrated with solvent A: $0.1 \%$ TFA in water. Solvent B: $0.05 \%$ TFA in MeCN. Gradient: $0-60 \%$ B in $45 \mathrm{~min}$. Flow rate: $1 \mathrm{ml} / \mathrm{min}$. The bar indicates TTR containing fractions.

acid substitution would require two nucleotide changes. Since no residues could be reliably confirmed beyond cycle 13 , the possibility of a frame shift was not excluded. Thus we repeated the purification and digestion of TTR from a second sampling of AP

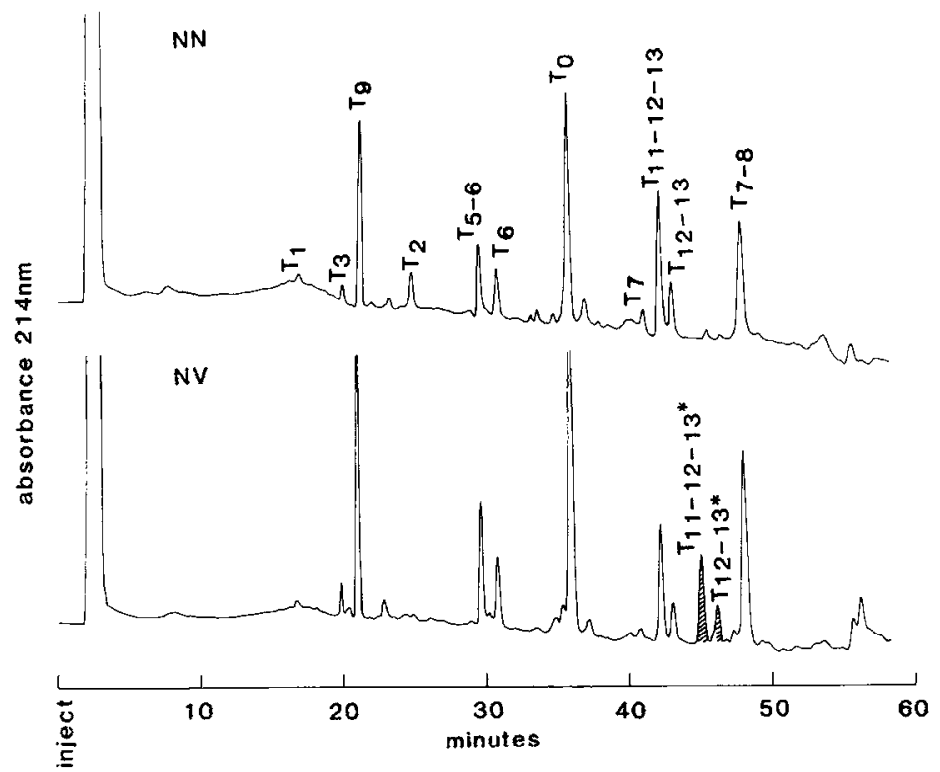

Figure 4. Reverse phase HPLC of TTR tryptic peptides. Column: C18 $\mu$ Bondapak equilibrated with solvent $A: 0.1 \%$ TFA in water. Solvent $B: 0.05 \%$ TFA in MeCN. Gradient: $0-60 \%$ B in $60 \mathrm{~min}$. Flow rate: $1 \mathrm{ml} / \mathrm{min}$. (Top) normal, NN, and (bottom) APTTR, NV, tryptic peptides. 
serum and isolated and sequenced the variant peptides. The second digestion was performed using more stringent digestion conditions to improve the yield of the variant peptide. TTR was denatured in 70\% formic acid (9), lyophilized and digested twice with trypsin (final E/S ratio=1:25). The variant peptides T11-12-13* and T12-13* were isolated for sequencing. Amino acid analysis indicated that the peptides consisted of residues 104 to 127 and 105 to 127 , respectively, the C-terminus. Microsequencing of T11-12-13* vielded positive identification of the amino acids in 16 of 18 cycles. The sequence, arg-tyr-thr-ile-ala-ala-leu-leu-ser-pro-tyr-ser-VAL-ser- ? - ? -ala-val(val,thr,asx,pro,lys,glu), was obtained. Residues in parentheses are from the amino acid composition and their order is inferred from the published sequence (18). The difference of a valine instead of tyrosine at cycle 13 was confirmed. A valine for tyrosine at residue 116 was also confirmed in peptide $T 12-13 \%$. In addition, serine, alanine and valine were confirmed at residues 117,120 and 121 , respectively, in agreement with the published sequence for normal TTR. The expected threonine residues at residues 118 and 119 could not be confirmed in either peptide T11-12-13* or T12-13*. The sequence and amino acid composition of the variant peptides thus exclude a frame shift as the mechanism of obtaining a valine for tyrosine at residue 116 .

The substitution of valine for tyrosine requires two nucleotide substitutions. Therefore we propose that the variant TTR arose from a mutation in a rare variant or heretofore undetected polymorphic allele of human TTR. There are two possibilities (phenylalanine or glutamic acid at residue 116) for the proposed "intermediate" allele. Glutamic acid at residue 116 would result in a polypeptide more anodal than normal TTR and which could focus in the position of the minor isoform of normal TTR (Figure 1). We have not detected such an anodal spot nor an increase in the intensity of the minor spot. Altland et al. (3) have detected a rare electrophoretic variant with a pI more acidic than normal TTR in one of 4,000 families studied. However, the amino acid substitution has not been determined. Alternatively, a neutral substitution of phenvlalanine for tyrosine could have gone undetected and could be a likely candidate for an undetected variant or polymorphic "intermediate".

The mechanism for the difference in mobility in the SDS dimension between the normal and variant TTR monomers is unclear. Slight differences in conformation or in the extent of SDS binding to the denatured polypeptide as a result of the substitution of valine for tyrosine could account for the mobility difference. Single amino acid substitutions in actin (19) and in hypoxanthine:guanine phosphoribosyl transferase (20) have also been shown to result in apparent molecular weight differences in the respective allelic polypeptides in SDS PAGE. 


\section{ACKNOWLEDGMENT}

This work was supported in part by NCI grant PO1-CA 26803.

\section{REFERENCES}

1. Rosenblum. B.B., Hanash, S.M. and Neel, J.V. (1983) In Proceedings, Fifth Intl. Prospective Biology Colloquium (G. Siest, M. Galteau and J. Henny, Eds.), pp. 85-90, Masson, Inc., New York.

2. Asakawa, J., Takahashi. N.. Rosenblum, B.B., and Neel, J.V. (1985) Hum. Genet. $70,222-230$.

3. Altland, K., Kast, Ch., Rauh. S., and Sgraja, Th. (1983) In Protides of the Biological Fluids (H. Peeters, Ed.). Vol. 30, pp 595-598. Pergamon Press, Oxford.

4. Lalloz, M.R.A., Bufield, P.G.H., Goel, K.M., Loudon. M.M., Thomson, J.A. and Himsworth. R.L. (1987) J. Clin. Endocrinol. Metab. 64, 346-352.

5. Nakazato, M. Kangawa, K.. Minamino. N., Tawara, S., Matsuo, H. and Araki, S. (1984) Biochem. Biophys. Res. Commun. 122, 712-718.

6. Saraiva, M.J., Birken. S.. Costa, P.P. and Goodman, D.S. (1984) J. Clin. Invest. 74 , 104-119.

7. Dwulet, F.E. and Benson, M.D. (1983) Biochem. Biophys. Res. Commun. 114, 657662.

8. Saraiva, M.J., Sherman, W. and Goodman, D.S. (1986) J. Lab. Clin. Med. 108, 1722.

9. Nakazato, M. Kangawa, K.. Minamino, N., Tawara. S., Matsuo, H. and Araki, S. (1984) Biochem. Biophys. Res. Commun. 123, 921-928.

10. Dwulet, F.E. and Benson. M.D. (1986) J. Clin. Invest. 78, 880-886.

11. Wallace, M.R., Dwulet, F.E., Conneally, P.M. and Benson. M.D. (1986) J. Clin. Invest. $78,6-12$.

12. Strahler, J.R., Rosenblum, B.B. and Hanash. S.M. (1983) J. Chromatogr. 266, 281291.

13. Rosenblum, B.B., Neel, J.V. and Hanash, S.M. (1983) Proc. Natl. Acad. Sci., USA 80, 5002-5006.

14. Merril, C.R.. Goldman. D.. Sedman, S.A. and Ebert. H. (1981) Science 211, 14371438.

15. Burnett, W.N. (1981) Anal. Biochem. 112, 195-203.

16. Boerwinkle. E.. Visvikis. S.. Strahler, J., Steinmetz, J. Hanash, S.M. and Sing. C.F. (1987) Am. J. Med. Genet. in press.

17. Tarr, G.E. (1986) In Methods of Protein Microcharacterization (J.E. Shively, Ed.). pp. 155-194. Humana Press, Inc.. Clifton, New Jersey.

18. Kanda, Y., Goodman, D.S., Canfield. R.E. and Morgan, F.J. (1974) J. Biol. Chem. 249. 6796-6805.

19. Vanderkerckhove, J.. Leavitt, J., Kakunaga, T. and Weber. K. (1980) Cell 22.893899.

20. Wilson, J.M.. Tarr, G.E. and Kelley, W.N. (1983) Proc. Natl. Acad. Sci., USA $\underline{80}$. 870-873. 